\title{
Robust Model Predictive Controller Design: Finite and Infinite Horizon
}

\author{
Vojtech Vesely, Daniel Vozak \\ Institute of Robotics and Cybernetics, Faculty of Electrical Engineering and Information Technology, Slovak University of \\ Technology in Bratislava, Ilkovicova 3, 81219 Bratislava.
}

Received: July 20, 2015 / Accepted: August 18, 2015 / Published: November 25, 2015.

\begin{abstract}
This paper addresses to the problem of designing, modeling and practical realization of robust model predictive control for finite and infinite prediction horizon which ensures a parameter dependent quadratic stability and guaranteed cost for linear polytopic uncertain systems. The model predictive controller design procedure based on BMI and LMI is reduced to off-line output feedback gain calculation. A numerical examples and an application to a real process is given to illustrate the effectiveness of the proposed method.
\end{abstract}

Keywords: MPC, robust stability, polytopic model, LMI, output feedback, infinite horizon

\section{Introduction}

Model predictive control (MPC) is a modern controller which is widely used in industrial applications. The main idea of MPC is to predict the future behavior of the system over a finite receding horizon and to find the optimal value of the system input with respect to predictions and given constraints. There exist several different realizations of MPC. Extensive overview of MPC algorithms can be found in Maciejowski [2002], Rossiter [2003].

The drawbacks of the most common MPC algorithms are the on-line computational complexity and the feasibility problem which in the past limited the use of MPC to plants with a slow time response because it solves optimization problem in each sample time. Another drawback when the plant model is uncertain or unstable is the lack of guaranteed stability and robustness of the closed-loop system that can cause a poor performance of the system. Numerous design procedures were developed to guarantee robust

Corresponding author: Vojtech Vesely, Institute of Robotics and Cybernetics, Faculty of Electrical Engineering and Information Technology, Slovak University of Technology in Bratislava. E-mail: vojtech.vesely@stuba.sk. stability. They are based on the infinite prediction or optimization horizon with the on-line LMI optimization [Kothare et al., 1996], the min-max optimization with a terminal constraint in an invariant set [Scokaert and Mayne, 1998, Lofberg, 2003], the off-line LMI optimization to calculate a sequence of output feedback laws and the on-line selection of the appropriate law [Ding et al., 2008] or the robust tube-based control design approach [Mayne et al., 2005]. The problems of the computational complexity and the robust stability are solved in the robust explicit MPC [Kerrigan and Maciejowski, 2004]. The dynamic output feedback robust model predictive controller for a system with both polytopic uncertainty and bounded disturbance is addressed in the paper [Ding, 2013]. This controller utilizes a main optimization problem to find the MPC control. An excellent survey of robust MPC design can be found in [Bemporad and Morari, 1999, Mayne et al., 2000, Rakovic, 2012]. Alternatively, in many implementations the MPC is applied without input and output constraints [Camacho and Bordons, 1999]. Then the algorithm reduces to linear feedback controller. But the design procedure still does not 
guarantee the stability or the robust stability and the closed-loop stability can be checked only by simulation. Recently the new MPC algorithm which guarantees the robustness of the closed-loop system have been presented in [Nguyen et al., 2013, Vesely and Rosinova, 2010, Vesely et al., 2010, Vozak and Vesely, 2014].

In this paper we pursue the ideas of [Vesely et al., 2010] where the robust MPC algorithm for finite prediction horizon has been designed on the base of the polytopic system quadratic stability and the input constraints, but the implementation problem in the real plants has been left out. For infinite prediction horizon we follow up the ideas of [Kothare et al., 1996] and the new MPC design procedure has been obtained. Hard input constraints can introduce to MPC using the approach [Vesely and Ilka, 2015]. However, the quadratic stability generally provides quite conservative results. To find a less conservative approach in this paper the parameter dependent quadratic stability (PDQS) [de Oliveira et al., 1999, Peaucelle et al., 2000] is used. The controller feedback gains calculation is formulated in the form of bilinear matrix inequalities (BMI). Because currently available BMI solvers do not allow to work with high order systems the problem of calculating an MPC output feedback gain needs to be transformed from BMI to LMI with a linearization approach. The designed controller solves the main drawbacks of the standard MPC because it guarantees the robust stability and requires only a simple on-line calculation based on the output feedback and the state estimation for a prediction model. The problem of MPC control algorithm design for the cases of finite and infinite prediction horizon using PDQS and its practical implementation is the main result of the paper.

The main principle of the controller design is described in the section 2 . In the section 3 and 4 an MPC design procedure without constraints is derived with its practical implementation. In the section 5 a new design procedure to MPC for the case infinite prediction horizon without constraints is derived. The last section contains a numerical example and a practical implementation to a real unstable magnetic levitation system with results from experiments. The following notational conventions will be adopted: The inequality $P>0 \quad(P<0)$ denotes the positive (negative) definiteness of the matrix, $I$ denotes the identity matrix with a corresponding dimension and the notation $y(k+h \mid k)$ is for simplicity reasons replaced by $y(k+h)$ and denotes a $h$ step ahead prediction of $y$ calculated in the sample time $k$.

\section{Preliminaries and Problem Formulation}

Let the polytopic linear discrete time system be described by

$$
\begin{aligned}
\tilde{x}(k+1) & =\tilde{A}(\xi) \tilde{x}(k)+\tilde{B}(\xi) u(k) \\
\tilde{y}(k) & =\tilde{C} x(k)
\end{aligned}
$$

where $\tilde{x}(k) \in R^{n}, \quad u(k) \in R^{m}, \quad \tilde{y}(k) \in R^{l}$ are state, control input and output variables of the system respectively. The matrices $\widetilde{A}(\xi)$ and $\widetilde{B}(\xi)$ belong to the convex set $S$, with $M$ vertices $S_{1}, \ldots, S_{M}$ that can be formally defined as:

$$
\begin{aligned}
S: & \left\{\widetilde{A}(\xi), \widetilde{B}(\xi):(\widetilde{A}(\xi), \widetilde{B}(\xi))=\sum_{i=1}^{M} \xi_{i}\left(\widetilde{A}_{i}, \widetilde{B}_{i}\right),\right. \\
& \left.\sum_{i=1}^{M} \xi_{i}=1, \xi_{i} \geq 0\right\}
\end{aligned}
$$

Matrices $\widetilde{A}_{i}, \widetilde{B}_{i}$ and $\widetilde{C}$ are known with constant coefficients of corresponding dimensions. Let consider the following integrator to force disturbance rejection and to achieve set-point tracking

$$
z(k+1)=z(k)-\widetilde{C} \widetilde{x}(k)+w(k)
$$

where $w(k)$ is a desired set-point value. Adding the integrator (3) to (1) one obtains:

$$
\begin{aligned}
x(k+1) & =A(\xi) x(k)+B(\xi) u(k)+B_{w} w(k) \\
y(k) & =C x(k)
\end{aligned}
$$

where 


$$
\begin{gathered}
x(k)=\left[\begin{array}{c}
\widetilde{x}(k) \\
z(k)
\end{array}\right], \quad A(\xi)=\left[\begin{array}{cc}
\widetilde{A}(\xi) & 0 \\
-\widetilde{C} & I
\end{array}\right], \quad B_{w}=\left[\begin{array}{l}
0 \\
I
\end{array}\right], \\
C=\left[\begin{array}{cc}
\widetilde{C} & 0 \\
0 & I
\end{array}\right], \quad B(\xi)=\left[\begin{array}{c}
\widetilde{B}(\xi) \\
0
\end{array}\right], \quad y(k)=\left[\begin{array}{l}
\widetilde{y}(k) \\
z(k)
\end{array}\right]
\end{gathered}
$$

$\tilde{y}(k)$ is a plant output for the proportional part of the controller and $z(k)$ is a plant output for the integral part of the controller. Optionally, the derivative part of the controller can be added in the form of the first difference:

$$
y_{d}(k)=\widetilde{y}(k-1)-\widetilde{y}(k)=\widetilde{C} \widetilde{x}(k-1)-\widetilde{C} \widetilde{x}(k)
$$

Then the system (4) is augmented as follows:

$$
\begin{gathered}
x(k)=\left[\begin{array}{c}
\widetilde{x}(k) \\
z(k) \\
z_{d}(k)
\end{array}\right], A(\xi)=\left[\begin{array}{ccc}
\widetilde{A}(\xi) & 0 & 0 \\
-\widetilde{C} & I & 0 \\
I & 0 & 0
\end{array}\right], B_{w}=\left[\begin{array}{c}
0 \\
I \\
0
\end{array}\right], \\
C=\left[\begin{array}{ccc}
\widetilde{C} & 0 & 0 \\
0 & I & 0 \\
-\widetilde{C} & 0 & \widetilde{C}
\end{array}\right], B(\xi)=\left[\begin{array}{c}
\widetilde{B}(\xi) \\
0 \\
0
\end{array}\right], y(k)=\left[\begin{array}{c}
\widetilde{y}(k) \\
z(k) \\
y_{d}(k)
\end{array}\right]
\end{gathered}
$$

The selection of the system (4) is based on (5) or (7) depending on the desired controller structure. Simultaneously with (4) we consider the nominal plant of the system in the form:

$$
\begin{aligned}
x(k+1) & =A_{0} x(k)+B_{0} u(k)+B_{w} w(k) \\
y(k) & =C x(k)
\end{aligned}
$$

where $A_{0}=\sum_{i=1}^{M} A_{i} \xi_{i 0}$ and $B_{0}=\sum_{i=1}^{M} B_{i} \xi_{i 0}$ for any values of $\xi_{i 0}$ which satisfy $\sum_{i=1}^{M} \xi_{i 0}=1, \quad \xi_{i 0} \geq 0$.

\subsection{Remarks}

(1) If the MPC design is based on the quadratic stability, the rate of change of coefficients $\xi_{i}$ may reach any value which satisfies (2).

(2) If the MPC design procedure is based on the parameter dependent quadratic stability, $\xi_{i}$ are constant and unknown.

(3) In the references [Vesely et al., 2010, Nguyen et al., 2013, Vesely and Rosinov'a, 2010], for the model predictive control design procedure there are two main ideas presented. In the first [Vesely et al., 2010] $\xi_{i 0}, i=1, \ldots, M$ are known and constant. In the last two references coefficients $\xi_{i 0}$ (for the realization of the prediction model) are known but may be changed.

(4) For the next we assume that the system model (4) and the nominal model (8) satisfy to remark 2.

The nominal model (8) will be used for the construction of the prediction model and (4) is considered as a real plant description providing the plant output. Therefore, we assume that for time $k$, output $y(k)$ is obtained from the uncertain plant model (4), and predicted outputs for time $k+1, \ldots, k+N_{y}$ will be obtained from the model prediction, when the nominal model (8) is used. The prediction is carried out over a finite output horizon $N_{y}$ and a control horizon $N_{u}\left(N_{u} \leq N_{y}\right)$. Note that we consider $F_{i j}=0$ for $i=N_{u}+1, \ldots, N_{y}$. Therefore for the next development we consider $N_{u}=N_{y}$.

Consider that for finite prediction horizon the predictive control algorithm with output feedback is as follows:

$$
\begin{aligned}
& u(k+i)=\sum_{j=0}^{N_{y}} \bar{F}_{i j}(\widetilde{y}(k+j)-w(k+j))+ \\
& \quad+E_{i j} z(k+j)=\sum_{j=0}^{N_{y}} F_{i j} y(k+j)-\bar{F}_{i j} w(k+j) \\
& \quad i=0,1, \ldots, N_{y}
\end{aligned}
$$

where $F_{i j}=\left[\begin{array}{ll}\bar{F}_{i j} & E_{i j}\end{array}\right], \quad \bar{F}_{i j} \in R^{m \times l}, \quad E_{i j} \in R^{m \times l} \quad$ are proportional and integral controller gain matrices, respectively.

States and outputs of the system for time instant $k$ are obtained from real system measurements:

$$
\begin{gathered}
x(k+1)=A(\xi) x(k)+B(\xi) u(k)+B_{w} w(k) \\
y(k)=C x(k)
\end{gathered}
$$

for time instants $k+h, h=1, \ldots, N_{y}$ the nominal model is used:

- $h=1$ 


$$
\begin{aligned}
& x(k+2)=A_{0} x(k+1)+B_{0} u(k+1) \\
& +B_{w} w(k+1) \\
& =A_{0} A(\xi) x(k)+A_{0} B(\xi) u(k)+B_{0} u(k+1) \\
& +A_{0} B_{w} w(k)+B_{w} w(k+1) \\
& y(k+1)=C x(k+1) \\
& \text { - } \vdots \\
& \text { - } h \\
& x(k+h+1)=A_{0}^{h} A(\xi) x(k)+\sum_{i=1}^{h} A_{0}^{h-i} B_{0} u(k+i)+ \\
& +A_{0}^{h} B(\xi) u(k)+\sum_{i=0}^{h} A_{0}^{h-i} B_{w} w(k+i) \\
& y(k+h)=C x(k+h)
\end{aligned}
$$

For $h=1, \ldots, N_{y}$ in the matrix form one obtains:

$$
\begin{aligned}
x_{f}(k+1) & =A_{f} x(k)+B_{f} v(k)+B_{w f} w_{f}(k) \\
y_{f}(k) & =C_{f} x_{f}(k)
\end{aligned}
$$

where

$$
\begin{gathered}
x_{f}(k)=\left[\begin{array}{c}
x(k) \\
\vdots \\
x\left(k+N_{y}\right)
\end{array}\right], \quad w_{f}(k)=\left[\begin{array}{c}
w(k) \\
\vdots \\
w\left(k+N_{y}\right)
\end{array}\right], \\
v(k)=\left[\begin{array}{c}
u(k) \\
\vdots \\
u\left(k+N_{y}\right)
\end{array}\right], \quad y_{f}(k)=\left[\begin{array}{c}
y(k) \\
\vdots \\
y\left(k+N_{y}\right)
\end{array}\right] \\
A_{f}=\left[\begin{array}{c}
A(\xi) \\
A_{0} A(\xi) \\
\vdots \\
A_{0}^{N_{y}} A(\xi)
\end{array}\right], C_{f}=\left[\begin{array}{cccc}
C & 0 & \ldots & 0 \\
0 & C & \ldots & 0 \\
\vdots & \vdots & \ddots & \vdots \\
0 & 0 & \ldots & C \\
B(\xi) & 0 & \ldots & 0 \\
A_{0} B(\xi) & B_{0} & \ldots & 0 \\
\vdots & \vdots & \ddots & \vdots \\
A_{0}^{N_{y}} B(\xi) & A_{0}^{N_{y}-1} B_{0} & \ldots & B_{0}
\end{array}\right], \\
B_{f}=\left[\begin{array}{cccc}
B_{w} & 0 & \ldots & 0 \\
A_{0} B_{w} & B_{w} & \ldots & 0 \\
A_{0}^{N_{y}} B_{w} & A_{0}^{N_{y}-1} B_{w} & \ldots & B_{w}
\end{array}\right]
\end{gathered}
$$

Matrices (14) in the system with prediction (12) are used only for calculation of robust controller gains (matrix $A_{f}$ is augmented with zeros to square matrix). In the practical implementation with respect to remark 4 matrices $A(\xi)$ and $B(\xi)$ are replaced with $A_{0}$ and $B_{0}$ when $\xi_{i 0}$ is constant. Then for the model prediction we obtain matrices $A_{f p}$ and $B_{f p}$ in the form:

$$
A_{f p}=\left[\begin{array}{c}
A\left(\xi_{0}\right) \\
A_{0}^{2} \\
\vdots \\
A_{0}^{N_{y}+1}
\end{array}\right] B_{f p}=\left[\begin{array}{cccc}
B\left(\xi_{0}\right) & 0 & \ldots & 0 \\
A_{0} B_{0} & B_{0} & \ldots & 0 \\
\vdots & \vdots & \ddots & \vdots \\
A_{0}^{N_{y}} B_{0} & A_{0}^{N_{y}-1} B_{0} & \ldots & B_{0}
\end{array}\right]
$$

If $\xi_{i 0}$ is known and time varying value then all matrices $A_{0}$ and $B_{0}$ in (8) should be recalculated in every sample time.

Control algorithm (9) can be rewritten to matrix form:

$$
v(k)=F y_{f}(k)-\bar{F} w_{f}(k)
$$

$$
\begin{aligned}
& F=\left[\begin{array}{cccc}
F_{00} & F_{01} & \ldots & F_{0 N_{y}} \\
F_{10} & F_{11} & \ldots & F_{1 N_{y}} \\
\vdots & \vdots & \ldots & \vdots \\
F_{N_{y} 0} & F_{N_{y} 1} & \ldots & F_{N_{y} N_{y}}
\end{array}\right] \\
& \bar{F}=\left[\begin{array}{cccc}
\bar{F}_{00} & \bar{F}_{01} & \ldots & \bar{F}_{0 N_{y}} \\
\bar{F}_{10} & \bar{F}_{11} & \ldots & \bar{F}_{1 N_{y}} \\
\vdots & \vdots & \ldots & \vdots \\
\bar{F}_{N_{y} 0} & \bar{F}_{N_{y} 1} & \ldots & \bar{F}_{N_{y} N_{y}}
\end{array}\right]
\end{aligned}
$$

Matrices $F_{i j}, i, j=0,1, \ldots, N_{y}$ are output feedback gains with constant entries to be determined by minimizing the cost function as follows:

$$
\begin{gathered}
J=\sum_{k=0}^{\infty} \widetilde{J}(k) \\
\widetilde{J}(k)=\sum_{j=0}^{N_{y}} x^{T}(k+j) q_{j} x(k+j)+\sum_{j=0}^{N_{u}} u^{T}(k+j) r_{j} u(k+j) \\
=x_{f}^{T}(k) Q x_{f}(k)+v^{T}(k) R v(k)
\end{gathered}
$$

$q_{j} \in R^{n \times n}, \quad r_{j} \in R^{m \times m}, Q$ and $R$ are positive definite matrices. 
The following lemmas will be used in the next development.

Lemma 1. Suppose there exists a Lyapunov function $V\left(x_{f}(k), \xi\right)$ such that for the first difference of a Lyapunov function

$\Delta V\left(x_{f}(k), \xi\right)=V\left(x_{f}(k+1), \xi\right)-V\left(x_{f}(k), \xi\right)$

with respect to (12) and control algorithm (9) the following inequality holds

$$
\Delta V\left(x_{f}(k), \xi\right)<0
$$

then the closed-loop system is robustly stable.

Lemma 2. Consider the system (4), if there exists a control law (9) and a Lyapunov function

$$
V\left(x_{f}(k), \xi\right)=x_{f}^{T}(k) P(\xi) x_{f}(k)
$$

such that the closed-loop system is robustly stable and for the first difference of a Lyapunov function on the closed-loop system solution holds:

$$
\Delta V\left(x_{f}(k), \xi\right)+\tilde{J}(k) \leq 0
$$

then the control law (9) is said to be the guaranteed cost control law with $J \leq J^{*}$.

Proof: Summing (20) from $k=k_{0}$ to $k \rightarrow \infty$ the upper bound on $J$ is obtained

$$
J \leq V\left(x_{f}\left(k_{0}\right), \xi\right)=J^{*}
$$

\section{MPC Design Procedure}

The design procedure is given as follows:

$$
\min _{F} J
$$

with constraints:

(1) System model (12) with given $N_{y}$ and $N_{u}$.

(2) Robust stability constraint and performance (20).

Note that in the above design procedure the optimisation results are the gain matrices $F_{i j}$, $i, j=0,1, \ldots, N_{y}$ which can be calculated off-line using the above design procedure which reduces to the standard robust controller design procedure. From [Peaucelle et al., 2000] the following lemma is obtained:
Lemma 3. Assume that the uncertain system with control algorithm is given by (12) and (15). The closed-loop system is parameter dependent quadratic stable if there exists a parameter dependent Lyapunov matrix $P(\xi)=\sum_{i=1}^{M} P_{i} \xi_{i}>0$, auxiliary matrices $H$ and $G$ such that the following inequality holds:

$$
\begin{aligned}
& {\left[\begin{array}{cc}
U_{1} & -H+A_{c i}^{T} G \\
-H^{T}+G^{T} A_{c i} & P-\left(G+G^{T}\right)
\end{array}\right]<0} \\
& U_{1}=-P_{i}+A_{c i}^{T} H^{T}+H A_{c i}+Q+C_{f}^{T} F^{T} R F C_{f} \\
& A_{c i}=A_{f i}+B_{f i} F C_{f}, \quad i=1,2, \ldots, M
\end{aligned}
$$

Because of BMI the following approach transforms above inequality to LMI. On the base of [Vesely et al. 2011] the two step LMI desing procedure is obtained:

(1) Solve the following matrix equations:

$$
\begin{aligned}
& A_{c i}^{T} P_{i} A_{c i}-P_{i}+Q+C_{f}^{T} F^{T} R F C_{f}<0 \\
& -P_{i}+A_{c i}^{T} H^{T}+H A_{c i}+Q+C_{f}^{T} F^{T} R F C_{f}<0
\end{aligned}
$$

where $Q$ and $R$ are weighting matrices in the cost function (17). Positive definite matrices $P_{i}$ and matrices $F, H$ represent unknown variables. Equations (24) are BMI. In order to transform (24) to linear matrix inequalities at first a Shur complement is used:

$$
\left[\begin{array}{ccc}
-P_{i}+Q & C_{f}^{T} F^{T} & \left(A_{f i}+B_{f i} F C_{f}\right)^{T} \\
F C_{f} & -R^{-1} & 0 \\
A_{f i}+B_{f i} F C_{f} & 0 & -P_{i}^{-1}
\end{array}\right]<0
$$

$$
\begin{aligned}
& {\left[\begin{array}{ccc}
U_{2} & C_{f}^{T} F^{T} & \left(H^{T}+B_{f i} F C_{f}\right)^{T} \\
F C_{f} & -R^{-1} & 0 \\
H^{T}+B_{f i} F C_{f} & 0 & -I
\end{array}\right]<0} \\
& U_{2}=-P_{i}+A_{f i}^{T} H^{T}+H A_{f i}-H H^{T}- \\
& -C_{f}^{T} F^{T} B_{f i}^{T} B_{f i} F C_{f}+Q
\end{aligned}
$$

Then a linearisation approach described in [Han and Skelton, 2003] transforms nonlinear diagonal terms to LMI by finding its upper bounds. Where for a given matrix $W$ the linearisation is:

$$
\operatorname{lin}\left(X^{-1}, X_{k}\right)=X_{k}^{-1}-X_{k}^{-1}\left(X-X_{k}\right) X_{k}^{-1}
$$




$$
\begin{aligned}
\operatorname{lin}\left(X W X, X_{k}\right)= & X_{k} W X_{k}-X W X_{k}- \\
& -X_{k} W X
\end{aligned}
$$

This linearisation approach requires several iterations to find a solution. At first an initial value for $X_{k}$ is selected. In the next iteration $X_{k}$ is equal to the calculated value of $X$ from the previous iteration.

(2) Check the robust stability. The LMI condition of parameter dependent quadratic stability [Peaucelle et al., 2000] is (23).

(3) If the solution is not feasible increase the eigenvalues of matrix $Q$ and goto step 1. In the step 2 the original matrix $Q$ is used.

For the next development assume that the gain matrices $F_{i j}$ are calculated off-line. The main problem is modeling and practical implementation of control algorithm (9) on the base of plant measurable output vectors $y(k)$ and states $x(k)$. Note that designed gain matrices $F_{i j}$ guarantee closed-loop stability, robustness and ensure guaranteed cost.

\section{Practical Implementation of MPC}

In this section we construct for a given $N_{y}$ and $N_{u}$ the final control algorithm for the case of robustly stable predictive control without input and output constraints.

Multiplying (10) from the left side by $C_{f}$ and substituting (15) to (10)

$$
\begin{aligned}
y_{f}(k)= & C_{f} A_{f} x(k)+C_{f} B_{f} F y_{f}(k)-C_{f} B_{f} \bar{F} w_{f}(k) \\
& +C_{f} B_{w f} w_{f}(k)
\end{aligned}
$$

Let matrix $C_{f} B_{f} F$ be partitioned as follows:

$$
C_{f} B_{f} F=\left[\begin{array}{ll}
M_{1} & \bar{M}_{2}
\end{array}\right]
$$

where $M_{1}$ is the first block column of matrix $C_{f} B_{f} F$.

$$
M_{1}=\left[\begin{array}{c}
C B_{0} F_{00} \\
C A_{0} B_{0} F_{00} \\
\vdots \\
C A_{0}^{N_{y}} B_{0} F_{00}
\end{array}\right]
$$

and $\bar{M}_{2}$ is the other part of matrix $C_{f} B_{f} F$. From (28) and (27) one obtains:

$$
\begin{aligned}
C_{f} B_{f} F y_{f}(k) & =M_{1} y(k)+\left[\begin{array}{ll}
\bar{M}_{2} & 0
\end{array}\right] y_{f}(k+1) \\
& =M_{1} y(k)+M_{2} y_{f}(k+1)
\end{aligned}
$$

Using (30) and (27):

$$
\begin{aligned}
{\left[I-M_{2}\right] y_{f}(k+1)=} & C_{f} A_{f} x(k)+M_{1} y_{f}(k) \\
& -C_{f}\left(B_{f} \bar{F}-B_{w f}\right) w_{f}(k)
\end{aligned}
$$

From (9) rewrite the control algorithm:

$$
\begin{aligned}
u(k)= & F_{00} y(k)+\left[\begin{array}{llll}
F_{01} & \ldots & F_{0 N_{y}} & 0
\end{array}\right] y_{f}(k+1)- \\
& -\left[\begin{array}{lll}
\bar{F}_{00} & \ldots & \bar{F}_{0 N_{y}}
\end{array}\right] w_{f}(k)
\end{aligned}
$$

Substituting (31) to (32) and after some manipulation:

$$
\begin{gathered}
u(k)=D_{1} x(k)+\left(F_{00}+D_{2}\right) y(k)-\left(D_{3}+\bar{F}_{0}\right) w_{f}(k) \\
D_{1}=\left[\begin{array}{llll}
F_{01} & \ldots & F_{N_{y}} & 0
\end{array}\right]\left[I-M_{2}\right]^{-1} C_{f} A_{f} \\
D_{2}=\left[\begin{array}{llll}
F_{01} & \ldots & F_{N_{y}} & 0
\end{array}\right]\left[I-M_{2}\right]^{-1} M_{1}
\end{gathered}
$$

$$
\begin{aligned}
& D_{3}=\left[\begin{array}{llll}
F_{01} & \ldots & F_{N_{y}} & 0
\end{array}\right]\left[I-M_{2}\right]^{-1} C_{f}\left(B_{f} \bar{F}-B_{w f}\right) \\
& \bar{F}_{0}=\left[\begin{array}{lll}
\bar{F}_{00} & \ldots & \bar{F}_{0 N_{y}}
\end{array}\right]
\end{aligned}
$$

Matrices $D_{1}, D_{2}, D_{3}$ for the case of $\xi_{i 0}=$ const. $, \quad i=1,2, \ldots, M$ are constant too and calculated off-line but for the case when $\xi_{i 0}$ changes, matrices $D_{1}, D_{2}, D_{3}$ should be recalculated in every sample time. Because gain matrices $F$ and $\bar{F}$ are constant and guarantee the closed-loop robust stability the calculation of $D$ matrices is straightforward. 


\section{Predictive Controller Design for Infinite Prediction Horizon}

In the seminal paper [Kothare et al., 1996] infinite prediction horizon has been proposed for model predicted state feedback controller design. In this part of paper the new design procedure for MPC output feedback is presented. For infinite prediction horizon $N_{y} \rightarrow \infty$ from (18) and (21) one obtains

$$
\begin{gathered}
\tilde{J}(k)=\sum_{j=0}^{\infty}\left(x^{T}(k+j) q_{j} x(k+j)+\right. \\
\left.u^{T}(k+j) r_{j} u(k+j)\right) \leq V(x(k), \xi)
\end{gathered}
$$

which gives the upper bound of cost function 18, [Kothare et al., 1996] when $N_{y} \rightarrow \infty$ and

$$
V(x(k), \xi)=x^{T}(k) X(\xi) x(k) X(\xi)=\sum_{i=1}^{M} X_{i} \xi_{i}>0
$$

For the infinite prediction horizon the predicted control design procedure (22) could be changed as follows

$$
\min _{\gamma} x^{T}(k) \gamma \operatorname{Ix}(k)
$$

with constraints

- System model (4)

- Stability and robust stability constraints (38)

$$
\Delta V(x(k), \xi)+\alpha V(x(k), \xi) \leq 0
$$

where the coefficient $0 \leq \alpha<1$ to influence on the closed-loop system performance has been introduced, and

$$
x^{T}(k) X(\xi) x(k) \leq x^{T}(k) \gamma I x(k)
$$

For the case of $N_{y} \rightarrow \infty$ the predicted output feedback control algorithm reduces to

$$
u(k)=K C x(k)
$$

where $K$ is output feedback gain matrix, and the closed loop system

$$
A_{c}(\xi)=A(\xi)+B(\xi) K C
$$

Equation (42) imply that for $x(k) \neq 0$ the following inequality holds

$$
X(\xi) \leq \gamma I
$$

or LMI solution

$$
\min _{\gamma}\left[\begin{array}{ll}
\gamma I & X_{j} \\
X_{j} & X_{j}
\end{array}\right] \geq 0 \quad j=1,2, \ldots M
$$

Note, that in [Kothare et al., 1996] original version the inequality (46) is in the form

$$
\min _{\gamma}\left[\begin{array}{cc}
\gamma & x^{T}(k) X_{j} \\
X_{j} x(k) & X_{j}
\end{array}\right] \geq 0 \quad j=1,2, \ldots M
$$

For practical realization at each sampling time one need to know the value of state variable $x(k)$ and then the output feedback gain matrix $K$ could be calculated using (47). For in the paper proposed case using the slightly modified inequalities (23) and (24) (only reduces the size) with (41) and (46) one obtain the inequalities to robust predicted controller design for infinite prediction horizon. The obtained model predicted controller for infinite prediction horizon practical implementation is straightforward.

\section{Experimental Results}

In order to prove applicability of the MPC algorithm two examples are presented. The first is a simulation of randomly generated system. In the second example the algorithm was implemented to control an unstable magnetic levitation system.

\subsection{Random System}

The system model is generated as a random discrete time model in the affine form:

$$
\begin{aligned}
& \tilde{A}(q)=\tilde{A}_{q 0}+q_{1} \tilde{A}_{q 1}+q_{2} \tilde{A}_{q 2} \\
& \tilde{B}(q)=\tilde{B}_{q 0}+q_{1} \tilde{B}_{q 1}+q_{2} \tilde{B}_{q 2}
\end{aligned}
$$

where $q_{1}, q_{2} \in\langle-1,1\rangle$.

$$
\begin{aligned}
& \tilde{A}_{q 0}=\left[\begin{array}{ll}
0.7060 & 0.2769 \\
0.0318 & 0.0462
\end{array}\right], \tilde{A}_{q 1}=\left[\begin{array}{ll}
0.1265 & 0.0557 \\
0.0195 & 0.1094
\end{array}\right] \\
& \tilde{A}_{q 2}=\left[\begin{array}{ll}
0.0656 & 0.0849 \\
0.0036 & 0.0934
\end{array}\right]
\end{aligned}
$$

$$
\tilde{B}_{q 0}=\left[\begin{array}{l}
0.6787 \\
0.7577
\end{array}\right] \tilde{B}_{q 1}=\left[\begin{array}{l}
0.7431 \\
0.3922
\end{array}\right] \tilde{B}_{q 2}=\left[\begin{array}{l}
0.6555 \\
0.1712
\end{array}\right]
$$

From (48) four vertices of polytopic model (1) are 
obtained:

$$
\begin{array}{ll}
\tilde{A}_{1}=\tilde{A}_{q 0}+\tilde{A}_{q 1}+\tilde{A}_{q 2}, & \tilde{B}_{1}=\tilde{B}_{q 0}+\tilde{B}_{q 1}+\tilde{B}_{q 2} \\
\tilde{A}_{2}=\tilde{A}_{q 0}-\tilde{A}_{q 1}+\tilde{A}_{q 2}, & \tilde{B}_{2}=\tilde{B}_{q 0}-\tilde{B}_{q 1}+\tilde{B}_{q 2} \\
\tilde{A}_{3}=\tilde{A}_{q 0}+\tilde{A}_{q 1}-\tilde{A}_{q 2}, & \tilde{B}_{3}=\tilde{B}_{q 0}+\tilde{B}_{q 1}-\tilde{B}_{q 2} \\
\tilde{A}_{4}=\tilde{A}_{q 0}-\tilde{A}_{q 1}-\tilde{A}_{q 2}, & \tilde{B}_{4}=\tilde{B}_{q 0}-\tilde{B}_{q 1}-\tilde{B}_{q 2}
\end{array}
$$

Calculated output feedback matrix $F$ with $Q=I$, $R=I, N_{y}=N_{u}=7$ and system model structure (5) is:

$$
F=\left[\begin{array}{ccccc}
-0.5525 & 0.3945 & -0.0141 & 0.0120 & -0.0057 \\
-0.6459 & 0.7151 & 0.0147 & 0.0070 & 0.0064 \\
-0.2396 & 0.2647 & 0.0024 & -0.0074 & 0.0011 \\
-0.1947 & 0.2148 & 0.0006 & -0.0104 & 0.0004 \\
-0.1964 & 0.2164 & 0.0004 & -0.0106 & 0.0003 \\
-0.2069 & 0.2279 & 0.0006 & -0.0104 & 0.0004 \\
-0.2076 & 0.2279 & 0.0005 & -0.0102 & 0.0003 \\
-0.0580 & 0.0589 & -0.0016 & -0.0051 & -0.0006
\end{array}\right.
$$

$\begin{array}{lllllll}0.0272 & -0.0028 & 0.0342 & -0.0009 & 0.0384 & 0.0007\end{array}$

$\begin{array}{llllll}-0.0079 & 0.0045 & -0.0145 & 0.0038 & -0.0192 & 0.0032\end{array}$

$\begin{array}{llllll}-0.0101 & 0.0005 & -0.0117 & -0.0000 & -0.0127 & -0.0005\end{array}$

$-0.0114-0.0001-0.0123-0.0006-0.0128-0.0010$

$-0.0115-0.0001-0.0123-0.0006-0.0128-0.0011$

$-0.0114-0.0001-0.0123-0.0006-0.0128-0.0010$

$-0.0111-0.0001-0.0119-0.0006-0.0124-0.0010$

$-0.0034-0.0004-0.0029-0.0003-0.0025-0.0003$

$\left.\begin{array}{lllll}0.0410 & 0.0025 & 0.0426 & 0.0070 & 0.0431\end{array}\right]$

$\begin{array}{lllll}-0.0234 & 0.0023 & -0.0273 & -0.0019 & -0.0309\end{array}$

$-0.0133-0.0009-0.0137-0.0018-0.0137$

$-0.0129-0.0015-0.0127-0.0019-0.0122$

$-0.0129-0.0015-0.0126-0.0019-0.0121$

$-0.0130-0.0014-0.0128-0.0019-0.0123$

$-0.0125-0.0014-0.0123-0.0017-0.0119$

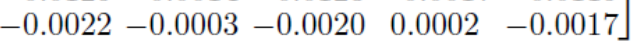

Matrix $\bar{F}$ is a proportional gain of the controller defined in (9). Then matrices (34)-(37) are:

$$
\begin{aligned}
& D_{1}=\left[\begin{array}{lll}
-0.0181 & -0.2541 & 0.2384
\end{array}\right] \\
& D_{2}=\left[\begin{array}{ll}
0.2942 & -0.2815
\end{array}\right] \\
& D_{3}=\left[\begin{array}{llll}
0.0558 & -0.2270 & -0.2003 & -0.1666
\end{array}\right. \\
& \left.\begin{array}{llll}
-0.1285 & -0.0874 & -0.0444 & -0.0005
\end{array}\right] \\
& F_{00}=\left[\begin{array}{ll}
-0.5525 & 0.3945
\end{array}\right] \\
& \bar{F}_{0}=\left[\begin{array}{lllll}
-0.5525 & -0.0141 & -0.0057 & -0.0028
\end{array}\right. \\
& \begin{array}{llll}
-0.0009 & 0.0007 & 0.0025 & 0.0070]
\end{array}
\end{aligned}
$$

Simulated system output and input is in Fig. 1.

\subsection{Magnetic Levitation}

The main principle of magnetic levitation model
CE152 made by HUMUSOFT [HUMUSOFT, 1996] is to control the position of a steel ball between a coil and an induction sensor. The process was identified in three working points (position $y=0.3,0.5,0.75[\mathrm{mu}]$ ) and a discrete-time polytopic model in the state-space with the sampling period $T_{s}=0.001$ was created:

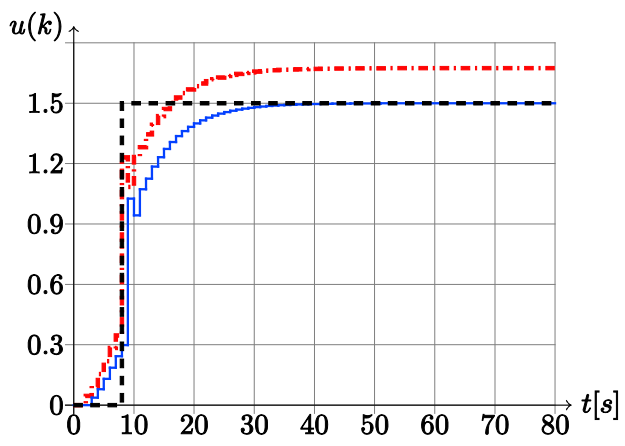

Fig. 1 Simulated system input (dash-dotted line), output (solid line) and set-point (dashed line).

$$
\begin{aligned}
& \tilde{A}_{1}=\left[\begin{array}{cc}
2.006 & 1 \\
-1.003 & 0
\end{array}\right] \tilde{B}_{1}=\left[\begin{array}{l}
0.00341 \\
0.00341
\end{array}\right] \\
& \tilde{A}_{2}=\left[\begin{array}{cc}
2.009 & 1 \\
-1.005 & 0
\end{array}\right] \tilde{B}_{2}=\left[\begin{array}{c}
0.0052 \\
0.00521
\end{array}\right] \\
& \tilde{A}_{3}=\left[\begin{array}{cc}
2.0 & 1 \\
-0.995 & 0
\end{array}\right] \tilde{B}_{3}=\left[\begin{array}{l}
0.00508 \\
0.00508
\end{array}\right] \\
& \tilde{C}=\left[\begin{array}{ll}
1 & 0
\end{array}\right]
\end{aligned}
$$

In order to find $F$ which is suitable for the application on real processes with respect to noise suppression and maximal values of manipulated variables it is possible to use additional constraints on $F$. Additional constraints easy to implemented using BMI or LMI solution. One of the possibilities is to constraint elements of $F=\left\{f_{i j}\right\}$ :

(1) $c_{1 \min } \leq f_{i j} \leq c_{1 \max } \quad$ for $j=1,4,7, \ldots, \quad \forall i$ (proportional part of the controller)

(2) $\quad c_{2 \min } \leq f_{i j} \leq c_{2 \max } \quad$ for $j=2,5,8, \ldots, \quad \forall i$ (integral part of the controller)

(3) $c_{3 \min } \leq f_{i j} \leq c_{3 \max }$ for $j=3,6,9, \ldots, \quad \forall i$ (derivative part of the controller)

The matrix $F$ calculated with $Q=1, R=85000$, $N_{y}=N_{u}=3$, and constraints $c_{1 \max }=-c_{1 \min }=5$, $c_{2 \max }=-c_{2 \min }=5$ and $c_{3 \max }=-c_{3 \min }=0.03$ is: 


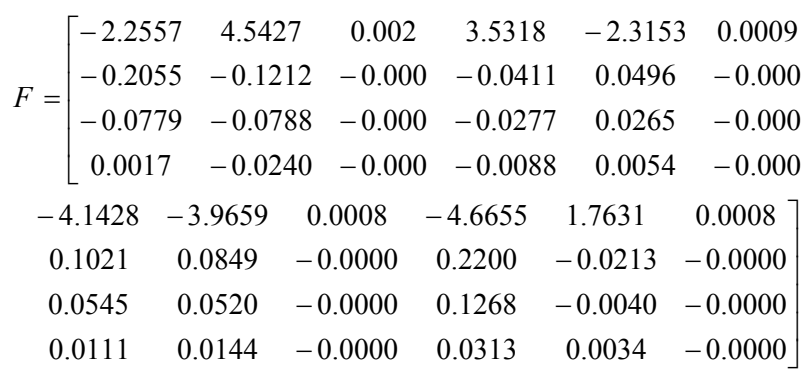

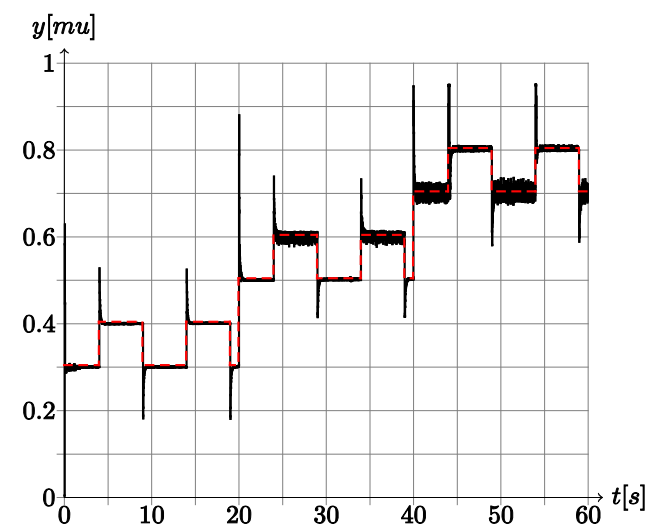

Fig. 2 Measured system output (solid line) and set-point (dashed line).

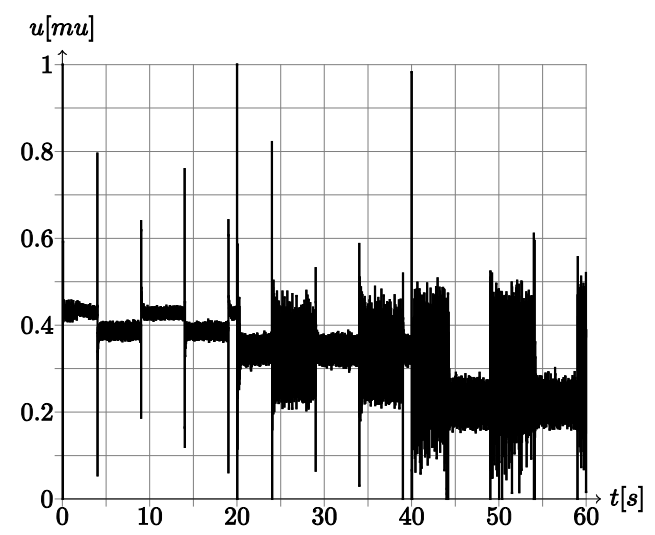

Fig. 3 Measured system input.

The equation of the observer is written as:

$$
\hat{x}(k+1)=\widetilde{A}_{0} \hat{x}(k)+\widetilde{B}_{0} u(k)+L \widetilde{C}(x(k)-\hat{x}(k))
$$

Observer gain matrix L was computed by solving the dual problem to the discrete-time LQ with weighting matrices $Q_{L}=10 I$ and $R_{L}=I$ of appropriate dimensions.

$$
L=\left[\begin{array}{c}
1.8542 \\
-0.9635
\end{array}\right]
$$

System output and input measured on real process experiments are in figures 2 and 3. The figures show that

- the system is robustly stable in all working points,

- the output has zero steady state error.

\section{Conclusion}

In the paper, new predictive control algorithms for the cases of finite and infinite prediction horizon guarantees the robust stability of the closed-loop system and the guaranteed cost control are presented. Due to off-line calculation of output feedback gain matrices its practical implementation is straightforward and with only simple on-line calculations required. In the case without input and output constraints it obviously reduces to linear feedback controller with constant gain matrices. For this approach a control algorithm was derived and practically implemented to control unstable magnetic levitation system. Obtained results prove good performance of the closed-loop system with MPC.

\section{References}

[1]. Bemporad and M. Morari.. Robustness in Identification and Control, 245:0 207-226, 1999.

[2]. E.F. Camacho and C.A. Bordons. Model Predictive Control. Springer-Verlag GmbH, 1999. ISBN 3540762418.

[3]. M.C. de Oliveira, J. Bernussou, and J.C. Geromel. A new discrete-time robust stability condition. Systems \& Control Letters, 370 (4):0 261-265, 1999. ISSN 0167-6911.

[4]. Baocang Ding, Yugeng Xi, Marcin T. Cychowski, and Thomas OMahony. A synthesis approach for output feedback robust constrained model predictive control. Automatica, 440 (1):0 258-264, 2008. ISSN 0005-1098.

[5]. Ding Baocang. New formulation of Dynamic output feedback robust model predictive control with guaranteed quadratic boundedness. Asian Journal of Control. Vol 15, 1, 302-309, 2013.

[6]. Jeongheon Han and R.E. Skelton. An LMI optimization approach for structured linear controllers. In Decision 
and Control, 2003. Proceedings. 42nd IEEE Conference, volume 5, pages 5143-5148 Vol.5, dec. 2003.

[7]. HUMUSOFT. Ce 152 magnetic levitation model, User's manual. 1996.

[8]. Eric C. Kerrigan and Jan M. Maciejowski. Feedback min-max model predictive control using a single linear program: robust stability and the explicit solution. International Journal of Robust and Nonlinear Control, 140 (4):0 395-413, 2004. ISSN 1099-1239.

[9]. Mayuresh V. Kothare, Venkataramanan Balakrishnan, and Manfred Morari. Robust constrained model predictive control using linear matrix inequalities. Automatica, 320 (10):0 1361-1379, 1996. ISSN 0005-1098.

[10]. J. Lofberg. Approximations of closed-loop minimax MPC. In Decision and Control, 2003. Proceedings. 42nd IEEE Conference on, volume 2, pages 1438-1442 Vol.2, 2003.

[11]. J. M. Maciejowski. Predictive control with constraints. Prentice Hall, 2002.

[12]. D.Q. Mayne, J.B. Rawlings, C.V. Rao, and P.O.M. Scokaert. Constrained model predictive control: Stability and optimality. Automatica, 360 (6):0 789-814, 2000.

[13]. D.Q. Mayne, M.M. Seron, and S.V. Rakovi. Robust model predictive control of constrained linear systems with bounded disturbances. Automatica, 410 (2):0 219-224, 2005. ISSN 0005-1098.

[14]. Quang Thuan Nguyen, Vojtech Vesely, and Danica Rosinova. Design of robust model predictive control with input constraints. International Journal of Systems
Science, 440 (5):0 896-907, 2013.

[15]. D. Peaucelle, D. Arzelier, O. Bachelier, and J. Bernussou.. Systems \& Control Letters, 40:0 21-30, 2000.

[16]. Sasa V. Rakovic. Invention of prediction structures and categorization of robust MPC syntheses. In 4th IFAC Nonlinear Model Predictive Control Conference, volume 4, pages 245-273, August 2012.

[17]. J.A. Rossiter. Model-Based Predictive Control: A Practical Approach. CRC Press Control Series. CRC Press, 2003. ISBN 9780849312915.

[18]. P. O M Scokaert and D.Q. Mayne. Min-Max feedback model predictive control for constrained linear systems. Automatic Control, IEEE Transactions on, 430 (8):0 1136-1142, 1998. ISSN 0018-9286.

[19]. Vojtech Vesely and Danica Rosinova. Robust output feedback model predictive control design. Archives of Control Sciences, 200 (3):0 253-266, 2010.

[20]. Vojtech Vesely, Danica Rosinova, and Martin Foltin.. ISA Transactions, 49:0 114-120, 2010.

[21]. Vojtech Vesely, Danica Rosinova, and Vladimir Kučera. Robust static output feedback controller LMI based design via elimination. Journal of the Franklin Institute, 3480 (9):0 2468-2479, 2011. ISSN 0016-0032.

[22]. Daniel Vozak and Vojtech Vesely. Robust model predictive controller design approach. In IFAC World COngress, August 2014, CD, 2014.

[23]. Vojtech Vesely and Adrian Ilka. Robust Controller Design with Input Constraints. Time Domain Approach. In IFAC, Symposium Rocond 2015, CD, 2015. 